\title{
PRINCIPALES MALESTARES BUCODENTALES PROVOCADOS POR CARIES DENTAL EN NIÑOS, CUENCA-ECUADOR, 2016
}

\section{MAIN BUCODENTALAL DISCOMFORT CAUSED BY DENTAL CARIES IN CHILDREN, CUENCA-ECUADOR, 2016}

\author{
Rosales-Bermeo Lourdes ${ }^{1 *}$ Encalada-Verdugo Liliana ${ }^{2}$ \\ ${ }^{1}$ Odontóloga egresada de la Universidad Católica de Cuenca. Ecuador \\ 2 Doctora en Odontología. Especialista en Docencia Universitaria. Mgs. en Estomatología, Docente de la Carrera de \\ Odontología de la Universidad Católica de Cuenca. Ecuador \\ *lencalada@ucacue.edu.ec
}

\begin{abstract}
Resumen
OBJETIVO. El objetivo de esta indagación fue establecer mediante la autopercepción, el impacto que causa la caries dental sobre los desempeños diarios en niños de 6 años de la parroquia Sucre, Cuenca, Ecuador, 2016. MATERIALES Y MÉTODOS: Es un estudio descriptivo retrospectivo donde se utilizaron las fichas epidemiológicas registradas en el departamento de investigación de la Universidad Católica de Cuenca, se analizo las fichas correspondientes a la PSCE, 2016, se seleccionó el cuestionario de SELF REPORT CARIES, y se procesaron los datos en el Epi Info. RESULTADOS: Se evaluo 177 niños de 6 años divididos en 103 de sexo femenino y 74 sexo masculino, pertenecientes a las escuelas de la parroquia Sucre, en donde se encontró que el impacto de la caries afectó a 152 estudiantes que representan un $86 \%$ de la muestra total, 90 del sexo femenino y 62 del sexo masculino. Los desempeños más afectados fueron comer $40 \%$ y lavarse los dientes $33 \%$; con respecto a su severidad, "Poco" fue el nivel de impacto reportado en mayor porcentaje. CONCLUSIÓN: Los desempeños diarios: comer y cepillarse fueron afectados en cada escolar.
\end{abstract}

Palabras clave: salud oral, calidad de vida, escolares..

\begin{abstract}
OBJECTIVE This inquiry was set up by self perception, the impact causes tooth decay on daily performance in 6 year children of Sucre, Cuenca, Ecuador, 2016. MATERIALS AND METHODS: A retrospective study in which the epidemiological records registered in the department of research of the University Catholic of Cuenca. First, the corresponding 2016 PSCE tabs were analyzed. Next, the questionnaire SELF REPORT was selected, and data were processed in the Epi info. RESULTS: 177 children were evaluated. The group was divided into 103 female and 74 male, belonging to schools in the Sucre parish, where it was found that the impact of caries affects to 152 students, representing $86 \%$ of the complete sample, 90 female and 62 male. The daily performances most affected were eating $40 \%$ and brushing teeth $33 \%$. However, according to their severity, "Little" was affected in a greater percentage. CONCLUSION: Daily performances: Eating and brushing were affected in each school.
\end{abstract}

Key words: oral health, quality of life, students..

\section{INTRODUCCIÓN}

Al citar la caries dental estamos hablando de una de los padecimientos más comunes en la boca de los niños. Se trata de una infección causada por bacterias, pero algunos factores están envueltos en este proceso, como son la desmineralización de los dientes, sangrado de encías, la presencia de placa bacteriana, los malos hábitos bucales, como la dieta mal llevada, y el consumo de medicamentos, que alteran el estado salud bucal. Las circunstancias de vida de cada individuo y el elevado costo de la atención dental se convierten en un gran inconveniente a nivel de la salud pública que puede incrementar el riesgo de caries. En la actualidad existen varios estudios a nivel mundial referentes a autopercepción que es la imagen que se hace el individuo cuando se trata de evaluar sus cualidades, es decir si se siente bien o si algo le incomoda. En la odontología este conocimiento, nos permite procesar 
dicha información desde el punto de vista clínico; pero a nivel del Ecuador existen pocos reportes en relación a el medio de salud bucal en nuestra comunidad. Por este motivo es necesario realizar estudios epidemiológicos descriptivos, con el fin de conocer la magnitud del problema de salud bucal en Cuenca. ${ }^{1}$ El presente estudio se realizó en las escuelas Ángel Polivio Chávez, San Roque, Remigio Romero y Cordero, Unidad Educativa Bilingüe y Eugenio Espejo pertenecientes a la parroquia Sucre del Cantón Cuenca, Ecuador, 2016.

El actual estudio está orientado al análisis de los datos obtenidos sobre la calidad de vida, la severidad de las caries y el impacto social que causa en los niños.

\section{MATERIALES Y MÉTODOS}

Se utilizó la ficha de recolección de información digital, en el programa de libre acceso EPI INFO v7.2; para registrar los datos que constan en las fichas epidemiológicas de la Universidad Católica de Cuenca, estas fichas constan de 5 partes: la primera de datos generales del paciente, la segunda de datos de Índice de Higiene Oral, la tercera de Índice de Caries CPOD, la cuarta de Índice de enfermedad Periodontal de Russel y la quinta de mal oclusiones.

La investigación se realizó entre los meses de septiembre y octubre del año 2016, recolectando datos de fichas epidemiológicas que reflejan la situación epidemiológica del año en curso. Dichas fichas fueron registradas entre los meses de mayo y julio del 2016. Para el análisis de los datos, se tomó en cuenta las fichas de la parroquia SUCRE, las cuales reflejan información epidemiológica con las siguientes características: El Estudio de autopercepción de caries dental buscó describir cualitativamente el problema en niños de 6 años de edad, utilizando los parámetros de la OMS, para diagnosticar presencia de caries dental, que se detalla a continuación. El procedimiento para utilizar el Niño-OIDP comenzó con un cuestionario auto administrado llevado a cabo con todos los niños en el aula. El cuestionario contiene una lista de todos los problemas orales que los niños son propensos a percibir y también incluyen una respuesta abierta para cualquier problema percibido inesperado. Se pidió a los niños a identificar los problemas orales que percibían en los últimos tres meses. Esta etapa tuvo como objetivo centrar la atención de los niños a sus problemas de salud oral y para llevar a la evaluación de impactos orales más tarde. Sus respuestas aquí se utilizan sólo como una guía para investigar los impactos orales sobre actuaciones diarias en el siguiente paso y se remitieron a cuando se les preguntó acerca de las causas de los impactos oral en entrevistas indi-viduales. A partir de entonces, los niños fueron entrevistados individualmente, con independencia de sus respuestas en el primer paso, para evaluar los impactos orales sobre la vida cotidiana en relación con 8 actuaciones diarias. Las 8 actua- ciones fueron: a) alimenticios, b) habla, c) limpieza de los dientes, d) relajante, incluyendo dormir, e) sonriendo, riendo y mostrando los dientes sin vergüenza, f) el mantenimiento de estudio el estado emocional, g), incluyendo ir a la escuela y haciendo) de contacto tarea y $\mathrm{h}$ con otras personas. Las en-trevistas individuales fueron complementadas por el empleo de 16 imágenes (imágenes negativas y positivas para cada actuación). Si los niños reportaron un impacto en cualquier actuación, se puntuaron la frecuencia de la incidencia y la gravedad de su efecto sobre su vida diaria. También se les pidió a los niños a identificar los problemas orales que, en su opinión, causaron el impacto.

Los problemas orales fueron identificados de la lista cumplido en el primer paso de la evaluación. ${ }^{26}$ La puntuación de impacto oral de cada rendimiento se obtiene multiplicando las puntuaciones de severidad y frecuencia, 0, 1, 2 o 3 cada uno, en relación a que el rendimiento.

\section{RESULTADOS}

Al analizar la muestra de 177 escolares se pudo evidenciar que 103 escolares fueron del sexo femenino (58\%) y 74 del sexo masculino (42\%). En relación con el tipo de gestión educativa 94 escolares pertenecieron a escuelas fiscales (53\%) y 83 a escuelas particulares $(47 \%)$. Se pudo demostrar que existió mayor número de población con impacto $(86 \%)$, y que la más afectada fue la población femenina (51 \%), también hubo mayor impacto en las escuelas fiscales (33 \% $19 \%$ ). Más sin embargo no hubo impacto de caries dental $(78 \%)$ sobre el desarrollo de los desempeños diarios pero si fue afectada la población de sexo femenino (14\%). Según la severidad, la caries afectó "poco" sobre los desempeños diarios (11\%) y según el tipo de escuela, la caries afecto más a las escuelas fiscales (15\%) sobre los desempeños diarios.

\begin{tabular}{lcccccc}
\hline & $\begin{array}{c}\text { FEMENINO } \\
\mathbf{n}\end{array}$ & $\%$ & $\begin{array}{c}\text { MASCULINO } \\
\mathbf{n}\end{array}$ & $\%$ & & $\%$ \\
\hline FISCAL & 59 & 33 & 35 & 20 & 94 & $53 \%$ \\
PARTICULAR & 44 & 25 & 39 & 22 & 83 & $47 \%$ \\
TOTAL & 103 & $58 \%$ & 74 & $42 \%$ & 177 & $100 \%$ \\
\hline
\end{tabular}

Tabla 1. Distribución de la muestra según el sexo y el tipo de escuela, en escolares de 6 años de la PSCE, 2016 


\begin{tabular}{ccccccc}
\hline & $\begin{array}{c}\text { CON } \\
\text { IMPACTO }\end{array}$ & $\%$ & $\begin{array}{c}\text { SIN } \\
\text { IMPACTO }\end{array}$ & $\%$ & TOTAL & $\%$ \\
\hline FEMENINO & $\mathbf{9 0}$ & $\mathbf{5 1 \%}$ & $\mathbf{1 3}$ & $7 \%$ & $\mathbf{1 0 3}$ & $\mathbf{5 8} \%$ \\
PARTICULAR & 32 & $18 \%$ & 12 & $7 \%$ & 44 & $25 \%$ \\
FISCAL & 58 & $33 \%$ & 1 & $1 \%$ & 59 & $33 \%$ \\
MASCULINO & 62 & $35 \%$ & 12 & $7 \%$ & $\mathbf{7 4}$ & $\mathbf{4 2} \%$ \\
PARTICULAR & 29 & $16 \%$ & 10 & $6 \%$ & 39 & $22 \%$ \\
FISCAL & 33 & $19 \%$ & 2 & $1 \%$ & 35 & $20 \%$ \\
TOTAL & 152 & $86 \%$ & 25 & $28 \%$ & 177 & $100 \%$ \\
\hline
\end{tabular}

Tabla 2. Impacto total de caries dental en niños de 6 años de la PSCE, 2016

\begin{tabular}{|c|c|c|c|c|c|c|}
\hline $\begin{array}{l}\text { DESEMPEN̈OS } \\
\text { DIARIOS }\end{array}$ & Total SI & $\%$ & Total NO & $\%$ & $\begin{array}{c}\text { Total } \\
\text { encuestados }\end{array}$ & $\%$ \\
\hline $\begin{array}{c}\text { COMER } \\
\text { ALIMENTOS }\end{array}$ & 71 & $40 \%$ & 106 & $60 \%$ & 177 & $100 \%$ \\
\hline CONTACTO & & & & & & \\
\hline CON OTRAS & 17 & $10 \%$ & 160 & $90 \%$ & 177 & $100 \%$ \\
\hline PERSONAS & & & & & & \\
\hline DESCANSAR & 19 & $11 \%$ & 158 & $89 \%$ & 177 & $100 \%$ \\
\hline $\begin{array}{l}\text { ESTADO DE } \\
\text { ANIMO }\end{array}$ & 37 & $21 \%$ & 140 & $79 \%$ & 177 & $100 \%$ \\
\hline $\begin{array}{c}\text { HABLAR } \\
\text { CLARAMENTE }\end{array}$ & 33 & $19 \%$ & 144 & $81 \%$ & 177 & $100 \%$ \\
\hline $\begin{array}{c}\text { LABORES } \\
\text { ESCOLARES }\end{array}$ & 28 & $16 \%$ & 149 & $84 \%$ & 177 & $100 \%$ \\
\hline $\begin{array}{l}\text { LAVARTE LOS } \\
\text { DIENTES }\end{array}$ & 58 & $33 \%$ & 119 & $67 \%$ & 177 & $100 \%$ \\
\hline $\begin{array}{l}\text { SONREIR, REIR, } \\
\text { MOSTRAR }\end{array}$ & 53 & $30 \%$ & 124 & $70 \%$ & 177 & $100 \%$ \\
\hline TOTAL & 316 & $22 \%$ & 1100 & $78 \%$ & 1416 & $100 \%$ \\
\hline
\end{tabular}

Tabla 3. Impacto de caries dental sobre los desempeños diarios en niños de 6 años de la PSCE, 2016 


\begin{tabular}{|c|c|c|c|c|c|c|}
\hline $\begin{array}{c}\text { DESEMPEÑOS } \\
\text { DIARIOS }\end{array}$ & FEMENINO $\mathrm{n}$ & $\%$ & MASCULINO n & $\%$ & Total SI & $\%$ \\
\hline $\begin{array}{c}\text { COMER } \\
\text { ALIMENTOS }\end{array}$ & 46 & $26 \%$ & 25 & $14 \%$ & 71 & $40 \%$ \\
\hline $\begin{array}{l}\text { CONTACTO CON } \\
\text { OTRAS PERSONAS }\end{array}$ & 8 & $5 \%$ & 9 & $5 \%$ & 17 & $10 \%$ \\
\hline DESCANSAR & 11 & $6 \%$ & 8 & $5 \%$ & 19 & $11 \%$ \\
\hline $\begin{array}{l}\text { ESTADO DE } \\
\text { ANIMO }\end{array}$ & 23 & $13 \%$ & 14 & $8 \%$ & 37 & $21 \%$ \\
\hline $\begin{array}{c}\text { HABLAR } \\
\text { CLARAMENTE }\end{array}$ & 22 & $12 \%$ & 11 & $6 \%$ & 33 & $19 \%$ \\
\hline $\begin{array}{c}\text { LABORES } \\
\text { ESCOLARES }\end{array}$ & 16 & $9 \%$ & 12 & $7 \%$ & 28 & $16 \%$ \\
\hline $\begin{array}{l}\text { LAVARTE LOS } \\
\text { DIENTES }\end{array}$ & 37 & $21 \%$ & 21 & $12 \%$ & 58 & $33 \%$ \\
\hline $\begin{array}{l}\text { SONREIR, REIR, } \\
\text { MOSTRAR }\end{array}$ & 39 & $22 \%$ & 14 & $8 \%$ & 53 & $30 \%$ \\
\hline Total general & 202 & $14 \%$ & 114 & $8 \%$ & 316 & $22 \%$ \\
\hline
\end{tabular}

Tabla 4. Impacto de caries dental sobre los desempeños diarios en niños de 6 años de la PSCE, 2016 según sexo 


\begin{tabular}{|c|c|c|c|c|c|c|c|c|}
\hline $\begin{array}{c}\text { DESEMPEÑOS } \\
\text { DIARIOS }\end{array}$ & POCO & $\%$ & REGULAR & $\%$ & MUCHO & $\%$ & $\begin{array}{c}\text { TOTAL } \\
\text { IMPACTOS }\end{array}$ & $\%$ \\
\hline $\begin{array}{c}\text { COMER } \\
\text { ALIMENTOS }\end{array}$ & 31 & $18 \%$ & 16 & $9 \%$ & 24 & $14 \%$ & 71 & $40 \%$ \\
\hline FEMENINO & 23 & $13 \%$ & 5 & $3 \%$ & 18 & $10 \%$ & & \\
\hline MASCULINO & 8 & $5 \%$ & 11 & $6 \%$ & 6 & $3 \%$ & & \\
\hline $\begin{array}{l}\text { CONTACTO CON } \\
\text { OTRAS PERSONAS }\end{array}$ & 10 & $6 \%$ & 6 & $3 \%$ & 1 & $1 \%$ & 17 & $10 \%$ \\
\hline FEMENINO & 3 & $2 \%$ & 4 & $2 \%$ & 1 & $1 \%$ & & \\
\hline MASCULINO & 7 & $4 \%$ & 2 & $1 \%$ & 0 & $0 \%$ & & \\
\hline DESCANSAR & 3 & $2 \%$ & 6 & $3 \%$ & 10 & $6 \%$ & 19 & $11 \%$ \\
\hline FEMENINO & 2 & $1 \%$ & 2 & $1 \%$ & 7 & $4 \%$ & & \\
\hline MASCULINO & 1 & $1 \%$ & 4 & $2 \%$ & 3 & $2 \%$ & & \\
\hline ESTADO DE ANIMO & 19 & $11 \%$ & 11 & $6 \%$ & 7 & $4 \%$ & 37 & $21 \%$ \\
\hline FEMENINO & 13 & $7 \%$ & 5 & $3 \%$ & 5 & $3 \%$ & & \\
\hline MASCULINO & 6 & $3 \%$ & 6 & $3 \%$ & 2 & $1 \%$ & & \\
\hline $\begin{array}{c}\text { HABLAR } \\
\text { CLARAMENTE }\end{array}$ & 19 & $11 \%$ & 8 & $5 \%$ & 6 & $3 \%$ & 33 & $19 \%$ \\
\hline FEMENINO & 13 & $7 \%$ & 5 & $3 \%$ & 4 & $2 \%$ & & \\
\hline MASCULINO & 6 & $3 \%$ & 3 & $2 \%$ & 2 & $1 \%$ & & \\
\hline $\begin{array}{l}\text { LABORES } \\
\text { ESCOLARES }\end{array}$ & 15 & $8 \%$ & 9 & $5 \%$ & 4 & $2 \%$ & 28 & $16 \%$ \\
\hline FEMENINO & 8 & $5 \%$ & 4 & $2 \%$ & 4 & $2 \%$ & & \\
\hline MASCULINO & 7 & $4 \%$ & 5 & $3 \%$ & 0 & $0 \%$ & & \\
\hline $\begin{array}{l}\text { LAVARTE LOS } \\
\text { DIENTES }\end{array}$ & 29 & $16 \%$ & 14 & $8 \%$ & 15 & $8 \%$ & 58 & $33 \%$ \\
\hline FEMENINO & 15 & $8 \%$ & 10 & $6 \%$ & 12 & $7 \%$ & & \\
\hline MASCULINO & 14 & $8 \%$ & 4 & $2 \%$ & 3 & $2 \%$ & & \\
\hline $\begin{array}{l}\text { SONREIR, REIR, } \\
\text { MOSTRAR }\end{array}$ & 28 & $16 \%$ & 15 & $8 \%$ & 10 & $6 \%$ & 53 & $30 \%$ \\
\hline FEMENINO & 18 & $10 \%$ & 14 & $8 \%$ & 7 & $4 \%$ & & \\
\hline MASCULINO & 10 & $6 \%$ & 1 & $1 \%$ & 3 & $2 \%$ & & \\
\hline Total general & 154 & $11 \%$ & 85 & $6 \%$ & 77 & $5 \%$ & 316 & $22 \%$ \\
\hline
\end{tabular}

Fig. 1. Severidad del impacto de caries dental sobre los desempeños diarios en niños de 6 años de la PSCE, 2016 según sexo

\begin{tabular}{ccccccc}
\hline $\begin{array}{c}\text { DESEMPEÑOS } \\
\text { DIARIOS }\end{array}$ & FISCAL & $\%$ & PARTICULAR & $\%$ & $\begin{array}{c}\text { Total } \\
\text { general }\end{array}$ & $\%$ \\
\hline $\begin{array}{c}\text { COMER } \\
\text { ALIMENTOS }\end{array}$ & 41 & $23 \%$ & 30 & $17 \%$ & 71 & $40 \%$ \\
$\begin{array}{c}\text { CONTACTO CON } \\
\text { OTRAS } \\
\text { PERSONAS }\end{array}$ & 14 & $8 \%$ & 3 & $2 \%$ & 17 & $10 \%$ \\
$\begin{array}{c}\text { DESCANSAR } \\
\text { ESTADO DE } \\
\text { ANIMO } \\
\text { HABLAR }\end{array}$ & 13 & $7 \%$ & 6 & $3 \%$ & 19 & $11 \%$ \\
$\begin{array}{c}\text { CLARAMENTE } \\
\text { LABORES }\end{array}$ & 24 & $16 \%$ & 9 & $5 \%$ & 37 & $21 \%$ \\
$\begin{array}{c}\text { ESCOLARES } \\
\text { LAVARTE LOS } \\
\text { DIENTES }\end{array}$ & 23 & $13 \%$ & 5 & $5 \%$ & 33 & $19 \%$ \\
$\begin{array}{c}\text { SONREIR, REIR, } \\
\text { MOSTRAR }\end{array}$ & 36 & $21 \%$ & 21 & $12 \%$ & 58 & $33 \%$ \\
Total general & $\mathbf{2 1 6}$ & $15 \%$ & 100 & $7 \%$ & 316 & $22 \%$ \\
\hline
\end{tabular}

Tabla 5. Impacto de caries dental sobre los desempeños diarios en niños de 6 años de la PSCE, 2016 según tipo de escuela. 


\begin{tabular}{|c|c|c|c|c|c|c|c|c|}
\hline $\begin{array}{l}\text { DESEMPEÑOS } \\
\text { DIARIOS }\end{array}$ & Poco & $\%$ & REGULAR & $\%$ & мUсно & $\%$ & $\begin{array}{l}\text { TOTAL } \\
\text { IMPACTOS }\end{array}$ & $\%$ \\
\hline $\begin{array}{l}\text { COMER } \\
\text { ALIMENTOS }\end{array}$ & 31 & $18 \%$ & 16 & $\mathbf{9 \%}$ & 24 & $14 \%$ & 71 & $40 \%$ \\
\hline FISCAL & 19 & $11 \%$ & 5 & $3 \%$ & 17 & $10 \%$ & & \\
\hline PARTICULAR & 12 & $7 \%$ & 11 & $6 \%$ & 7 & $4 \%$ & & \\
\hline $\begin{array}{l}\text { CONTACTO CON } \\
\text { OTRAS } \\
\text { PERSONAS }\end{array}$ & 10 & $6 \%$ & 6 & $3 \%$ & 1 & $1 \%$ & 17 & $10 \%$ \\
\hline FISCAL & 8 & $5 \%$ & 5 & $3 \%$ & 1 & $1 \%$ & & \\
\hline PARTICULAR & 2 & $1 \%$ & 1 & $1 \%$ & 0 & $0 \%$ & & \\
\hline \multirow{3}{*}{$\begin{array}{l}\text { DESCANSAR } \\
\text { FISCAL } \\
\text { PARTICULAR }\end{array}$} & 3 & $2 \%$ & 6 & $3 \%$ & 10 & $6 \%$ & 19 & $11 \%$ \\
\hline & 1 & $1 \%$ & 4 & $2 \%$ & 8 & $5 \%$ & & \\
\hline & 2 & $1 \%$ & 2 & $1 \%$ & 2 & $1 \%$ & & \\
\hline \multirow{2}{*}{$\begin{array}{l}\text { ESTADO } \\
\text { ANIMO } \\
\text { FISCAL }\end{array}$} & 19 & $11 \%$ & 11 & $6 \%$ & 7 & $4 \%$ & 37 & $21 \%$ \\
\hline & 14 & $8 \%$ & 9 & $5 \%$ & 5 & $3 \%$ & & \\
\hline PARTICULAR & 5 & $3 \%$ & 2 & $1 \%$ & 2 & $1 \%$ & & \\
\hline \multirow{3}{*}{$\begin{array}{l}\text { HABLAR } \\
\text { CLARAMENTE } \\
\text { FISCAL } \\
\text { PARTICULAR }\end{array}$} & 19 & $11 \%$ & 8 & $5 \%$ & 6 & $3 \%$ & 33 & $19 \%$ \\
\hline & 14 & $8 \%$ & 6 & $3 \%$ & 4 & $2 \%$ & & \\
\hline & 5 & $3 \%$ & 2 & $1 \%$ & 2 & $1 \%$ & & \\
\hline $\begin{array}{l}\text { LABORES } \\
\text { ESCOLARES }\end{array}$ & 15 & $8 \%$ & 9 & $5 \%$ & 4 & $2 \%$ & 28 & $16 \%$ \\
\hline FISCAL & 14 & $8 \%$ & 6 & $3 \%$ & 3 & $2 \%$ & & \\
\hline PARTICULAR & 1 & $1 \%$ & 3 & $2 \%$ & 1 & $1 \%$ & & \\
\hline \multirow{3}{*}{$\begin{array}{l}\text { LAVARTE LOS } \\
\text { DIENTES } \\
\text { FISCAL } \\
\text { PARTICULAR }\end{array}$} & 29 & $16 \%$ & 14 & $8 \%$ & 15 & $8 \%$ & 58 & $33 \%$ \\
\hline & 18 & $10 \%$ & 12 & $7 \%$ & 7 & $4 \%$ & & \\
\hline & 11 & $6 \%$ & 2 & $1 \%$ & 8 & $5 \%$ & & \\
\hline \multirow{2}{*}{$\begin{array}{l}\text { SONREIR, REIR, } \\
\text { MOSTRAR } \\
\text { FISCAL }\end{array}$} & 28 & $16 \%$ & 15 & $8 \%$ & 10 & $6 \%$ & 53 & $30 \%$ \\
\hline & 16 & $9 \%$ & 12 & $7 \%$ & 8 & $5 \%$ & & \\
\hline PARTICULAR & 12 & $7 \%$ & 3 & $2 \%$ & 2 & $1 \%$ & & \\
\hline Total general & 154 & $11 \%$ & 85 & $6 \%$ & 77 & $5 \%$ & 316 & $22 \%$ \\
\hline
\end{tabular}

Tabla 6. Severidad del impacto de caries dental sobre los desempeños diarios en niños de 6 años de la PSCE, 2016 según tipo de escuela.

\section{DISCUSIÓN}

El presente estudio fue realizado en la parroquia Sucre en la provincia del Azuay, está localizada en el centro de la ciudad de Cuenca, los niños de este estudio pertenecen a la clase media, se la denomina así ya que se encuentran cerca del centro de la urbe. Como la principal fuente de ingresos de los padres de las escuelas fiscales tenemos el comercio ya que dichos miembros de la familia realizan labores relacionadas con el mismo, mientras que los padres de las escuelas particulares ejercen profesiones diversas.

El número de la población perteneciente a este estudio fue de 177 niños de 6 años de edad, lo cual discrepa del estudio de Encalada.13 ya que este estudio fue realizado en una muestra de 35 escolares en el año 2015, todo esto se corrobora ya que ella realiza un estudio piloto en la provincia del Cañar en escuelas urbanas y rurales. Sin embargo concuerda en relación con la investiga-ción de Del Castillo.16 con una muestra de estudio de 150 escolares en el año 2010, de Paredes.26 con 169 en 2013 y con 132 de Ingunza12 en 2015, en distintos lugares del Perú incluyendo los sectores urbanos, rurales y marginales, esto garantiza resultados más acertado ya que estos estudios fueron elaborados como trabajos de tesis.

El impacto de caries sobre los desempeños diarios primer lugar para el desempeño comer alimentos que alcanzó un porcentaje del $40 \%$ de afección a nivel de la niñez, esto se compara con el estudio de Paredes.? Realizado en Perú que alcanza los valores de $55 \%$ de afección. Ahora, al hablar según el sexo y el tipo de gestión educativa, los desempeños más afectados fueron comer y lavarse los dientes en la pobla- población femenina y las escuelas fiscales. Coincidiendo así la afección entre los desempeños diarios y el sexo. Ahora, al analizar según la severidad del impacto sobre los desempeños diarios el mayor porcentaje estuvo sobre el nivel "poco"; siendo afectado el desempeño comer alimentos con el $18 \%$, que según el sexo y el tipo de gestión educativa que afectó más al sexo femenino y a las escuelas fiscales, estos datos se relacionan con el estudio de Encalada. ${ }^{1,2}$ Que obtuvo valores de severidad para el nivel poco en un $38 \%$ pero que en este afectó más a los niños de escuelas rurales, además el segundo desempeño más afectado fue lavarse los dientes (16 $\%)$ que afectó de igual manera al sexo masculino como femenino pero afectó más a las escuelas fiscales; esto se relaciona con el estudio de Paredes. ${ }^{26}$ en donde el desempeño lavarse los dientes también estuvo en segundo lugar pero afectó más al sexo masculino. Lo cual puede ser por que la población en este estudio fue en mayor porcentaje sobre la población femenina mientras que el estudio de Paredes tuvo mayor población de sexo masculino, pero sigue coincidiendo el desempeño y la gestión académica.

\section{CONCLUSIÓN}

Con el presente estudio se determinó la manera en cómo afecta la caries sobre los desempeños diarios, ya que cada escolar tuvo al menos un desempeño limitado. Se estimó la severidad del impacto de caries sobre los desempeños diarios, el sexo y el tipo de escuela, como "poco", afectando en mayor número a la población femenina y a la población de las escuelas fiscales. 


\section{Referencias}

1 Informe de la Academia Americana de Pediatría, Medida para el Riesgo en el Tiempo de Salud Oral y Establecimiento del Hogar Dental. PEDIATRICS Volumen 111 No 5 Mayo 2003, pp 1113-1116. En el Internet en http://aappolicy.aappublications. org/cgi/reprint/pediatrics; $111 / 5 / 1113$ Disponible en: documento disponible.

2 Vidal Gutiérez D, Chamblas García I, Zavala Gutiérrez M, Muller Gilchrist R, Rodríguez Torres M, Chávez Montecino A. Determinantes sociales el salud y estilos de vida en la población, 2014 Disponible en: documento disponible.

3 Cléa A. Saliba-Garbin, Artênio J. Isper-Garbin, Renato Moreira-Arcieri, Nemre A Saliba y Patricia E. Gonçalves, la salud bucal en la percepción del adolecente, 2009 Disponible en: documento disponible.

4 OMS. Salud bucodental abril 2012, Disponible en: documento disponible.

5 Moyaho-Bernal Angeles, Lara-Muñoz Ma. Carmen, Espinosa-De Santillana Irene, Muñoz-Quintana Gabriel, Etchegoyen Graciela. La auto-percepción de la salud general y bucal en los niños de Puebla, México.

6 Narváez Trujillo H. Determinantes sociales y su relación con caries en niños y niñas de 1 a 5 años de la fundación "Niños de María" de la ciudad de Quito durante el año lectivo 2011-2012", 2015. Disponible en: documento disponible.

7 Gonzáles Penagos C, Cano Gómez M, Meneses Gómez E, Vivares Builes A.(2015) "Percepciones en salud bucal de los niños y niñas"

8 OMS. La atención primaria de salud, 2008 Disponible en: documento disponible.

9 Morales González J, Nava Chapa G, Esquivel Soto J, Díaz Pérez L. Principios de ética, bioética y conocimiento del hombre, 2011. Disponible en: documento disponible.

10 Meriñán S. Las enfermedades dentales en niños afectan su desempeño, 2013. documento disponible.

11 Ingunza J, Del Castillo López C, Evangelista Alva A, Sánchez Borja P. Calidad de vida relacionada a la salud bucal en escolares de ámbito urbano marginal, Lima, 2013 Disponible en: documento disponible.

12 Encalada Verdugo L. Estudio Piloto Comparativo de salud oral relacionada a la calidad de vida en escolares del cantón Cañar-Ecuador, 2015. Disponible en: documento disponible.

13 Pitiphat W, García RI, Douglass CW, Joshipura KJ (2002) Validación de las medidas de salud oral de auto-reporte. J Public Health Dent.

14 Moyaho Bernal y colaboradores. La autopercepción de la salud general y bucal en los niños de Puebla México. 2010. Disponible en: documento disponible.
15 Del Castillo López y colaboradores. Impacto de las condiciones bucales sobre la calidad de vida en escolares de ámbito rural, Piura, Perú, 2014 Disponible en: documento disponible.

16 Quintanilla Díaz de León D, Determinación del índice de higiene oral en niños de 6 a 12 años en el área metropolitana de Monterrey, Nuevo León en el año 2011

17 Guitelman I, Mondello A, Cortese Silvina G, Biondi A. Autopercepción y salud bucal en niños. Disponible en: documento disponible.

18 Reinoso Vintimilla N, Del Castillo C. Caries dental y problemas orales auto percibidos en escolares de 12 años de Sayausí, Cuenca, Ecuador. Rev Estomatol Herediana. 2017 Oct-Dic;27(4):227-34 Disponible en: documento disponible.

19 Fernández Gonzalez, Constanza Núñez F, María L. (Prof. Guía).Estado de salud oral y percepción de calidad de vida en niños de 12 años, de área rural y urbana, que asisten a algunos establecimientos municipalizados de las comunas de Talca, Maule, Pelarco y San Clemente, 2008

20 Villavicencio Caparó E, Research Gate. [Online]. Arequipa; 2010 [cited 2016104 . Available from: documento disponible.

21 Villavicencio Caparó E., Sayago-Heredia J, Katherine C, Velez-León E, Cabrera Duffaut A. Pasos para la planificación de una investigación clínica. Odontología Activa. 2016;: p. 73-75. documento disponible.

22 Ilustre Municipalidad de Cuenca. Ordenanza de división de las parroquias urbanas de la Ciudad de Cuenca. Ordenanza \#74 (4). 1982. Disponible en: documento disponible.

23 Adulyanon S, Sheiham A. Impactos orales sobre su Vida Diaria. En Medición de la salud oral y la calidad de vida .Editado por: Slade GD. Chapel Hill: Universidad de Carolina del Norte; 1997:. 151-160 Disponible en: documento disponible.

24 Jokovic A, D Locker Stephens M, Kenny D, Tompson B, Guyatt G, Validez y fiabilidad de un cuestionario para medir infantil de calidad relacionadas con la salud oral de la vida. J Dent Res 2002, 81:. 459-463 DISPONIBLE EN: documento disponible.

25 Gherunpong S, El desarrollo de un sistema socio-dental de la evaluación de las necesidades dentales en los niños de la tesis doctoral. University College de Londres, Departamento de Epidemiología; 2004. Disponible en: documento disponible.

26 Paredes Martínez E, Impacto de las condiciones bucales sobre la calidad de vida en escolares del distrito de San Juan de Miraflores. Lima. 2014. Disponible en documento disponible.

Recibido: 29 de enero de 2018. 